University of Nebraska - Lincoln

DigitalCommons@University of Nebraska - Lincoln

May 1993

\title{
Nanostructural effects and interface magnetism in Co/Pd multilayers
}

Ping $\mathrm{He}$

University of Nebraska - Lincoln

Z.-S. Shan

University of Nebraska - Lincoln

John A. Woollam

University of Nebraska-Lincoln, jwoollam1@unl.edu

David J. Sellmyer

University of Nebraska-Lincoln, dsellmyer@unl.edu

Follow this and additional works at: https://digitalcommons.unl.edu/physicssellmyer

Part of the Physics Commons

He, Ping ; Shan, Z.-S.; Woollam, John A.; and Sellmyer, David J., "Nanostructural effects and interface magnetism in Co/Pd multilayers" (1993). David Sellmyer Publications. 110.

https://digitalcommons.unl.edu/physicssellmyer/110

This Article is brought to you for free and open access by the Research Papers in Physics and Astronomy at DigitalCommons@University of Nebraska - Lincoln. It has been accepted for inclusion in David Sellmyer Publications by an authorized administrator of DigitalCommons@University of Nebraska - Lincoln. 


\title{
Nanostructural effects and interface magnetism in Co/Pd multilayers
}

\author{
Ping He, ${ }^{\text {a) }}$ Z. -S. Shan, ${ }^{\text {b) John A. Woollam, }}$, and D. J. Sellmyer ${ }^{\text {b) }}$ \\ University of Nebraska, Lincoln, Nebraska 68588-0511
}

\begin{abstract}
A series of $\mathrm{Co} / \mathrm{Pd}$ multilayers were made by de magnetron sputter deposition on $\mathrm{Al}$ foil substrates. For these multilayered samples, Co layer thicknesses were less than $4 \AA$ and $\mathrm{Pd}$ layers were varied from 4 to $22 \AA$. Sputtcring rates were controlled by either sputtering power $(10-50 \mathrm{~W})$ or Ar sputtering pressure (3-15 mTorr). In both cases, lower deposition rates yielded higher perpendicular coercivity up to $2.6 \mathrm{kOe}$. Structures of the samples were studied using conventional $\theta-2 \theta$ x-ray diffractometry (XRD). It has been found that magnetic properties such as coercivity and saturation magnetization are sensitive to interfacial structures. A nanostructural model including interfacial parameters such as alloy layer composition is discussed and compared with the magnetization data. Both XRD and magnetization measurements show that the interfaces become more diffuse at higher sputtering pressures.
\end{abstract}

\section{INTRODUCTION}

Multilayered magnetic materials have become an active research area ${ }^{1,2}$ due to the new magnetic properties that are suitable for variety of applications such as magneto-optic (MO) recording. The new properties include large perpendicular anisotropy and high coercivity that often do not exist in single-layer films. For the application to magneto-optic recording media, $\mathrm{Co} / \mathrm{Pt}$ and $\mathrm{Co} / \mathrm{Pd}$ have been intensively investigated for several years. ${ }^{3-7}$ These two material systems are being considered for second generation $\mathrm{MO}$ recording media.

Because these magneto-optic multilayered systems consist of ultrathin material layers, it is likely that the thickness of interfaces and alloy composition at interfaces play a crucial role in the new magnetic and magnetooptical properties. In this study, we investigated interfacial structures and magnetism, including spin polarization of Pd atoms, for samples deposited in different conditions. A simple model was employed to relate quantities such as film composition and bilayer thickness to interfacial structures. Therefore, magnetic properties of $\mathrm{Co} / \mathrm{Pd}$ multilayers can be better understood.

\section{EXPERIMENT}

Samples were sputter deposited onto water-cooled Al foil substrates under various sputtering conditions. Ar sputtering gas pressure was controlled by a mass flow meter. Multilayered structures were deposited by rotation of the substrates above the guns, and the time when a substrate stopped above either Co or Pd gun was controlled so that a desired amount of material was deposited. A total of 16 multilayer samples was prepared with different layered structures or different deposition conditions. According to different deposition conditions and structures, the multilayered samples can be divided into three groups, and the nominal layered structure and magnetic properties are summarized in Tables I and II.

\footnotetext{
"Center for Microelectronic and Optical Material Research.

${ }^{6}$ Center for Materials Research and Analysis.
}

The layered and crystalline structures of these samples were studied by $x$-ray difractometry (XRD). Both smalland large-angle scans were made in the conventional $\theta-2 \theta$ scan mode. Magnetic properties, such as coercivity and magnetization, were measured on an alternating gradient force magnetometer (AGFM). The film composition of samples was determined by measuring the mass of each Co and Pd deposited, and was also verified by energy dispersive spectroscopy (EDS).

\section{RESULTS AND DISCUSSIONS}

Samples in groups I and II show the magnetic properties as a function of different deposition conditions, such as sputtering power of $\mathrm{Pd}$ gun for samples in group I and Ar sputtering pressure for samples in group II. The direct effect of changing sputtering power or sputtering pressure is to change the material deposition rate. All the samples in groups I and II show perpendicular hysteresis loops with squareness near 1 , but the coercivity of these samples varies significantly. It can be seen from Fig. 1 that lower deposition rate caused by either lower sputtering power or higher sputtering pressure results in higher coercivity $H_{c}$ determined form hysteresis loops. Since the deposition rate

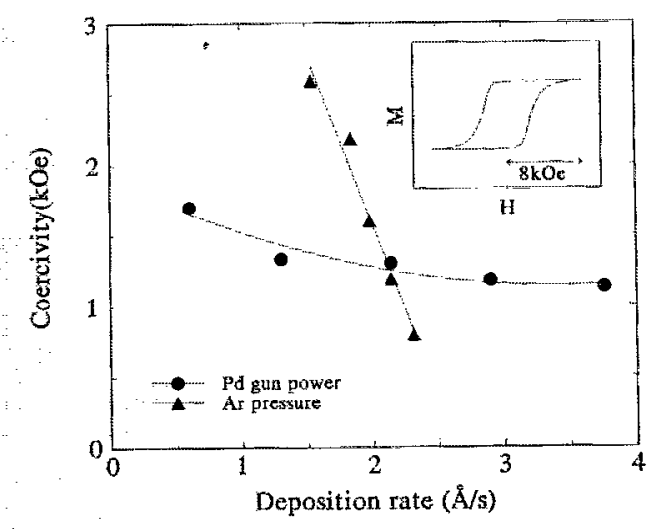

FIG. 1. Coercivity $H_{c}$ vs deposition rates for samples in groups I and II. The deposition rate was changed by either sputtering power or sputtering pressure. The same deposition rate does not yield the same $H_{i^{*}}$ The inset is a typical perpendicular $\mathbf{M}-H$ loop (from sample $[\mathbf{I}-5$ ). 
TABLE L. Sputtering power (group I) and sputtering pressure (group) effects on magnetism and interfaces. Nominal layered structure: $\mathrm{Co}(2$ A) $\operatorname{Pd}(13$ A $\times 35$.

\begin{tabular}{|c|c|c|c|c|c|}
\hline \multicolumn{6}{|c|}{ Group I (Co gun power $=30 \mathrm{~W}$, sputtering pressure $=6$ mTorr $)$} \\
\hline Sample No. & I-1 & $\mathrm{I}=2$ & $\mathrm{I}-3$ & $\mathrm{I}-4$ & $\mathrm{I}-5$ \\
\hline \multicolumn{6}{|l|}{ Pd gun power } \\
\hline$(W)$ & 10 & 20 & 30 & 40 & 50 \\
\hline$H_{i}(\mathrm{Oe})$ & 1700 & 1330 & 1300 & 1180 & 1130 \\
\hline$g_{y}(\mathrm{emu} / \mathrm{g})$ & 226 & 228 & 224 & 228 & 226 \\
\hline \multicolumn{6}{|c|}{ Group II $\left(\mathrm{Co}_{4}\right.$ Pd gun power $\left.=30 \mathrm{~W}\right)$} \\
\hline Sample No. & $\mathrm{II}=1$ & $\mathrm{II}-2$ & $. \mathrm{II}-3$ & II-4 & $\mathrm{II} \times 5$ \\
\hline Sputter pressure & & & & & \\
\hline (mTorr) & 3 & 6 & 9 & 12 & 15 \\
\hline $\mathrm{H}_{\mathrm{e}}(\mathrm{Oe})$ & 792 & 1190 & 1600 & 2180 & 2590 \\
\hline$\sigma_{s}(\mathrm{emu} / \mathrm{g})$ & 218 & 201 & 206 & 191 & 187 \\
\hline
\end{tabular}

changes more significantly with the change of sputtering power rather than sputtering pressure, the sputtering power is the main factor that changes the sputtering yield ${ }^{8}$ and deposition rate. However, the change of $H_{c}$ due to the change of sputtering power is modest. From the point of view of dynamic molecular theory, one atom on the surface will experience about 300 collisions per second with atoms of the gas when the pressure is about $1 \mathrm{~m}$ Torr. Therefore, the chamber ambient during film deposition plays an important role in the deposition process and has significant effects on the film quality. Therefore, the sputtering pressure is the major factor affecting $H_{c}$ as shown in Fig. 1.

Small-angle XRD for samples in group I shows qualitatively similar peaks (which are not shown) in the sense that both the peak position and intensity do not vary much. As shown in Table $I$, the saturation magnetizations $\sigma_{s}$, calculated from measured value of $M-H$ hysteresis loop and Co mass, of samples in group I do not show any significant difference between them, suggesting that interfacial magnetism of these samples is basically the same. However, the intensity of the (111) peaks and small-angle peaks from samples in group II shows systematic changes with sputtering pressure, as shown in Fig. 2. Higher-order small-angle peaks and other large-angle peaks are not observed for samples in group II. It can be seen that when the sputtering pressure is greater than $9 \mathrm{~m}$ Torr, the smallangle peak disappears, indicating more diffused interfaces or lack of well-defined layer boundaries, which may be caused by the frequent collisions between surface atoms and ambient gas atoms. The saturation magnetization $\sigma_{s}$ of

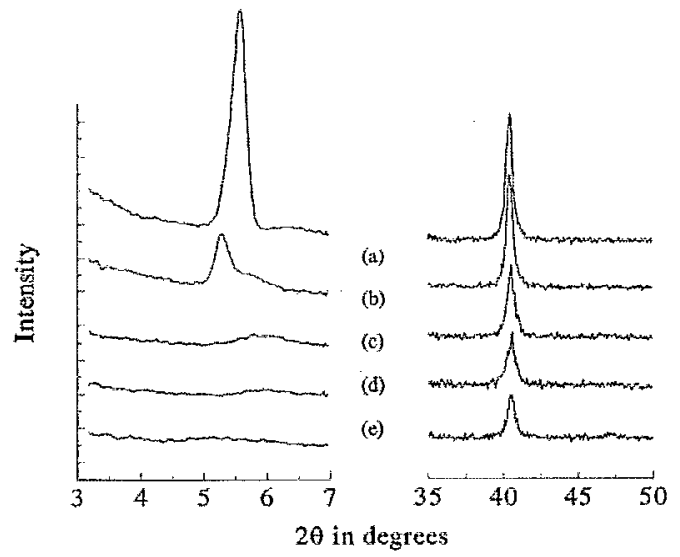

FIG. 2. Large- and small-angle XRD scans on samples in group II. The peaks on large-angle scans are (111) peaks. The peak changes show the interdiffusion dependence on different sputtering pressure. Diffraction patterns from top to bottom: (a) $3 \mathrm{mTorr}$, (b) $6 \mathrm{mTorr}$, (c) $9 \mathrm{~m}$ Torr, (d) $12 \mathrm{mTorr}$, and (e) $15 \mathrm{mTorr}$.

samples in group II exhibits a systematic decrease with an increase of sputtering pressure, as well. Since, as revealed by small-angle XRD of these samples, the higher the sputtering pressure, the more diffuse the interfaces, the decrease of $\sigma_{s}$ may be caused by either the decrease of induced magnetic moments on $\mathrm{Pd}$ atoms, or the reduction of Curie temperature. As Shan et al. pointed out, ${ }^{9}$ the induced moments on $\mathrm{Pd}$ are a function of the number of nearest $\mathrm{Co}$ neighbors around $\mathrm{Pd}$ atoms. The fewer the Co neighbors around $\mathrm{Pd}$ atoms, the less the induced moments on Pd atoms. It should be noticed that more Pd atoms will be polarized because more $\mathrm{Pd}$ atoms are mixed with $\mathrm{Co}$ atoms due to the interdiffusion, which will increase the magnetization of the film. These two results have opposite effects on the overall magnetization $\sigma_{s}$. The results listed in Tables I and II show that the decrease of induced moments on $\mathrm{Pd}$ is dominant. The other explanation of the decrease of induced moments is that when the interfaces are more diffuse, the Curie temperature $T_{c}$ will decrease which can cause the decrease in magnetization. According to $\mathrm{Co}-\mathrm{Pd}$ thermal equilibrium phase diagram, $T_{c}$ is about room temperature at 90 at. $\%$ Pd.

The magnetization $\sigma_{s}$ of samples in group III is shown in Fig. 3. It can be seen that the initial increase of $\sigma_{s}$ is due to the induced moment on $\mathrm{Pd}$ atoms which are surrounded

TABLE II. Pd layer thickness effect on interface magnetism. Co layer thickness of $2 \hat{\AA}$ was fixed. Bilayer thickness was determined by small-angle XRD, and film composition by EDS.

\begin{tabular}{|c|c|c|c|c|c|c|}
\hline \multirow[b]{2}{*}{ Sample No. } & \multicolumn{5}{|c|}{ Group III (nominal structure: $\operatorname{Co}(2 \AA) / \operatorname{Pd}(x \AA) \times 35)$} & \multirow[b]{2}{*}{ III-6 } \\
\hline & 111-1 & III-2 & III -3 & III-4 & III-5 & \\
\hline$x(A)$ & 4.0 & 7.0 & 10.0 & 13.0 & 16.0 & 22.0 \\
\hline$H_{e}(\mathrm{Oe})$ & $744.0^{*}$ & $817.0^{a}$ & 1040.0 & 1190.0 & 1210.0 & 1010.0 \\
\hline$\sigma_{s}(\mathrm{emu} / \mathrm{g})$ & 202.0 & 229.0 & 249.0 & 246.0 & 245.0 & 249.0 \\
\hline Bilayer thick. $(A)$ & 6.5 & 10.1 & 13.1 & 15.5 & 18.9 & 24.9 \\
\hline Film conp. ${ }^{1 /}(\mathrm{Co})$ & 0.37 & 0.24 & 0.18 & 0.15 & 0.13 & 0.10 \\
\hline
\end{tabular}

aquareness is less than 0.9.

"The film composition is in atomic percent of $\mathrm{Co}$. 


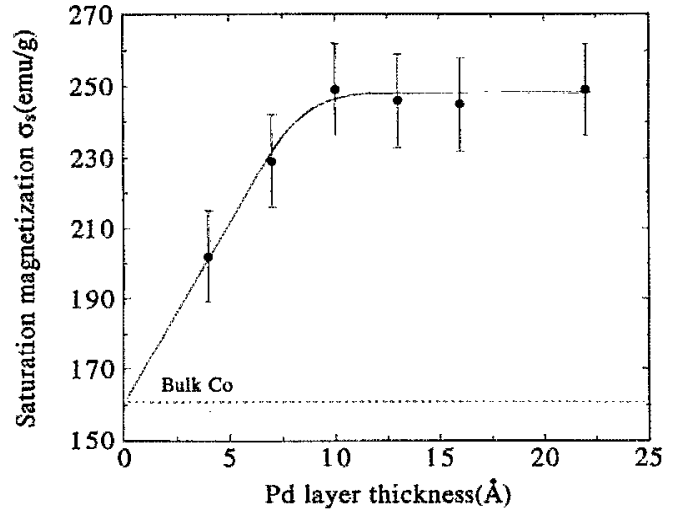

FIG. 3. Saturation magnetization $\sigma_{s}$ as function of Pd layer thickness for samples of group III. $\sigma_{\xi}$ is calculated using Co mass only. The major source of the error bars is from the measurement of Co mass in the multiayered films.

or partially surrounded by Co atoms. The shape of the curve in Fig. 3, in general, agrees with data obtained by others. ${ }^{10}$ One difference found in this study is that $\sigma_{\xi}$ (Fig. 3) stays roughly constant after $\sigma_{5}$ reaches a maximum at a Pd layer thickness of $\sim 10 \AA$, indicating that Pd atoms added after the Pd layer is greater than $10 \AA$ make no contribution to the magnetization, and thus these Pd atoms are not mixed with $\mathrm{Co}$, as is reasonable. Therefore, if it is assumed that $\mathrm{Co}$ atoms diffuse in both directions into adjacent Pd layers, the interface thickness will be about $10 \AA$. The interdiffusion length is then estimated to be about $5 \AA$.

More information about Co-Pd interfaces may be obtained when magnetic, XRD, and EDS measurements are combined. A microstructural model ${ }^{11}$ can be used to estimate the average composition of Co at interfaces or in the CoPd alloy layers. This model can be expressed as the following:

$$
L=L_{a}\left(1-\frac{n_{a}}{n_{b}}\right)+\frac{n_{a}}{n_{b}} L_{a} \frac{x_{\mathrm{Co}}^{a}}{x_{\mathrm{Co}}},
$$

where $L$ is the bilayer thickness which can be measured by small-angle XRD, $L_{a}$ the alloy layer (interface) thickness, $n_{a}$ and $n_{b}$ the atomic number densities of the alloy layers and pure Pd layers, and $x_{\mathrm{Co}}^{a}$ and $x_{\mathrm{Co}}$ (determined by EDS or other methods) the Co compositions in the alloy layers and in the film, respectively.

The bilayer thickness and over all Co composition of these samples in group III can be easily and accurately determined and are listed in Table II. The plot of $L$ vs $1 / x_{\mathrm{Co}}$ shows a straight line in Fig. 4. From the slope $(=2.45 \AA)$, intercept $(=-0.314 \AA)$, and alloy layer thickness $(=2 \times$ interdiffusion length $=10-12 \AA$ ) which is estimated above, the average Co composition at the interfaces is found to be about $20-25$ at. \%. Also, the atomic number ratio $n_{a} / n_{b}$ can be calculated as 1.03 which is com-

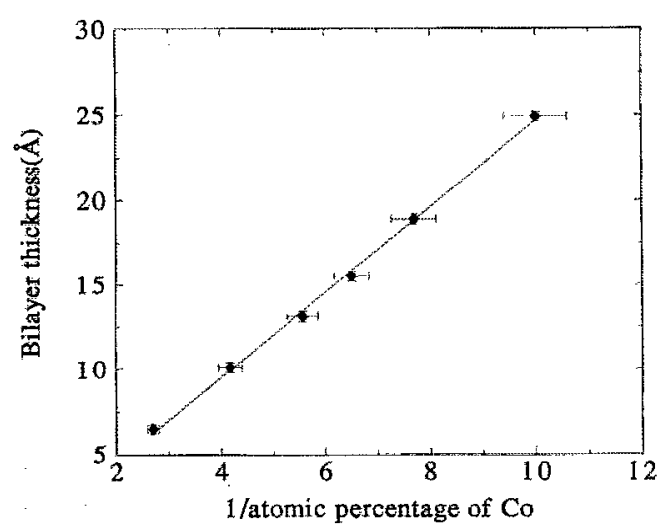

FIG. 4. Bilayer thickness $L$ vs $1 / x_{C o}$, where $x_{C o}$ is the overall Co composition of films for samples in group III.

parable to the value of about 1.06 for bulk $\mathrm{Co}_{0.2} \mathrm{Pd}_{0.8}$ alloy and bulk Pd. From the above discussion, it can be seen that although the model is very simple, it allows one to calculate reasonably correct microstructural parameters and to obtain a detailed picture of interfaces.

In summary, sputter-deposited $\mathrm{Co} / \mathrm{Pd}$ multilayers were studied. We found that the interdiffusion and changes in $H_{c}$ and $\sigma_{s}$ when sputtering pressure increases are directly related to interaction between surface atoms and ambient gas. Deposition rate has little cffect on these magnetic properties. Nanostructures at interfaces have been discussed in detail. Co composition at interfaces has been calculated to be about $20-25$ at. \% for samples deposited at $6 \mathrm{mTorr}$.

\section{ACKNOWLEDGMENTS}

The authors want to thank the National Science Foundation for supporting the research at the University of Nebraska, grant No. DMR-891889. We also thank Dr. S. Nafis for valuable discussions.

'See, for example, Science and Technology of Nanostructured Magnetic Materials, edited by G. C. Hadjipanayis and G. A. Prinz (Plenum, New York, 1991).

${ }^{2}$ L. M. Faclicov et at, J. Mater. Res. 5, 1299 (1990).

${ }^{3}$ S. Klahn, P. Hansen, and F. J. A. M. Greidanus, Vacuum 41, 1160 (1990).

${ }^{4}$ S. Hashimoto, Y. Ochiai, and K. Aso, J. Appl. Phys. 66, 4909 (1989).

${ }^{5}$ P. He, W. A. MeGahan, S. Nafis, J. A. Woollam, S. H. Liou, F. Sequeda, T. McDaniel, and H. Do, J. Appl. Phys. 70, 6044 (1991).

${ }^{6}$ P. He, W. A. McGahan, J. A. Woollam, F. Sequeda, T. MeDaniel, and H. Do, J. Appl. Phys. 69, 4021 ( 1991 ).

'P. F. Carcia, S. I. Shah, and W. B. Zeper, Appl. Phys. Lett. 56, 2345 (1990).

${ }^{8}$ R. V. Stuart, Vacuum Technology, Thin Films, and Sptuttering (Academic, New York, 1983).

${ }^{9}$ Z. S. Shan, P. He, C. Moore, J. A. Woollam, and D. J. Sellmyer, J. Appl. Phys. (to be published)

${ }^{10}$ D. G. Stinson and S. C. Shin, J. Appl. Phys, 67, 4459 (1990).

${ }^{11} \mathrm{P}$. He and J. A. Woollam, The Proceedings of 41 st Annual Denver $X$-ray Conference, Colorado Springs, CO, August 3-7, 1992. 